\title{
A Case of Hidden Foreign Body: How We did It?
}

\author{
${ }^{1}$ Miria Mathews, ${ }^{2}$ Roshna Rose Paul, ${ }^{3}$ George Ani Mathew, ${ }^{4}$ Rita Ruby Albert
}

\begin{abstract}
Pediatric age groups commonly present with history of fish or chicken bone getting stuck in throat while eating. They usually present early due to odynophagia and the usual site is the cricopharynx. This is a case of chicken bone getting stuck in the posterior pharyngeal wall of a child which presented late and caused quite a dilemma to remove it. This report is to highlight the unusual site of foreign body and the use of fluoroscopy to remove it.
\end{abstract}

Keywords: Chicken bone, Fluoroscopy, Foreign body.

How to cite this article: Mathews M, Paul RR, Mathew GA, Albert RR. A Case of Hidden Foreign Body: How We did It? Int J Otorhinolaryngol Clin 2015;7(2):75-77.

\section{Source of support: Nil}

\section{Conflict of interest: None}

\section{INTRODUCTION}

Foreign body ingestion is common in pediatric age group. It is seen mostly in the age group 3 months to 1 year. Seventy percent of foreign bodies ingested are coins. ${ }^{1}$ Other common foreign bodies include vegetable, such as pea, fishbone, chicken bone, parts of toy and battery. Batteries, sharp pieces of metal objects or bone are especially harmful since they can perforate. Though most of the ingested foreign body passes through into the stomach without obstructing the food passage, a small percentage can get impacted at different sites of anatomical narrowing along the upper aerodigestive tract. $^{2}$ The most common site of impaction for fish bone is usually at the level of the tonsils, although may be found at the base of the tongue, the vallecula or the pyriform fossa. ${ }^{3}$ Cricopharyngeal sphincter is the most common site for other foreign bodies. ${ }^{4}$ In older children, ingestion is almost always accidental. Migration of foreign body is quite rare and mostly seen with fish bone which is later found in the lateral neck. ${ }^{5}$ We report a case of 5 days old migratory foreign body in a child and its management.

\footnotetext{
${ }^{1}$ Postgraduate, ${ }^{2}$ Assistant Professor, ${ }^{3}$ Associate Professor ${ }^{4}$ Professor

${ }^{1-4}$ Department of ENT, Christian Medical College, Vellore, Tamil Nadu, India

Corresponding Author: Roshna Rose Paul, Assistant Professor, Department of ENT, Christian Medical College Vellore, Tamil Nadu, India, Phone: 04162286089, e-mail: anhsor@gmail.com
}

\section{CASE REPORT}

A 7-year-old child presented to pediatric emergency department with history of chicken bone ingestion 5 days back, followed by complaints of throat pain and inability to swallow solid food for 3 days. There was no history of fever, noisy breathing or voice change. He was admitted in a local hospital, where he underwent diagnostic esophagoscopy twice under general anesthesia, but was unsuccessful in the foreign body removal. He was referred to our hospital for further management.

The child was comfortable during examination. His vitals were stable and neck appeared normal. On palpation, there was tenderness over right side of the neck. X-ray soft tissue neck anteroposterior and lateral views (Fig. 1) were done, which showed foreign body at the level of $\mathrm{C} 3-\mathrm{C} 4$ with minimal widening of prevertebral soft tissue.

He underwent diagnostic direct laryngoscopy with esophagoscopy, but foreign body could not be visualized nor palpated. There was no edema or raw mucosa in the posterior pharyngeal wall. There was mucosal abrasion and a deep pocket seen in the left pyriform fossa with minimal pus within it. We used zero degree telescope into the pocket to visualize but no foreign body was seen. It was postulated that, the pocket was probably made during the previous esophagoscopy. It was then decided to insert a Ryle's tube, abort procedure and to take him up after computed tomography (CT) imaging to confirm position of the foreign body. Computed tomography neck with oral contrast (Figs 2A and B) was done which showed the foreign body to be at C3-C4 vertebral level but in the retropharyngeal space.

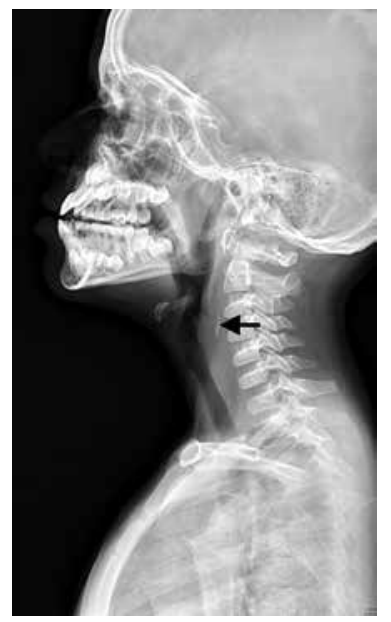

Fig. 1: Plain radiograph neck lateral 

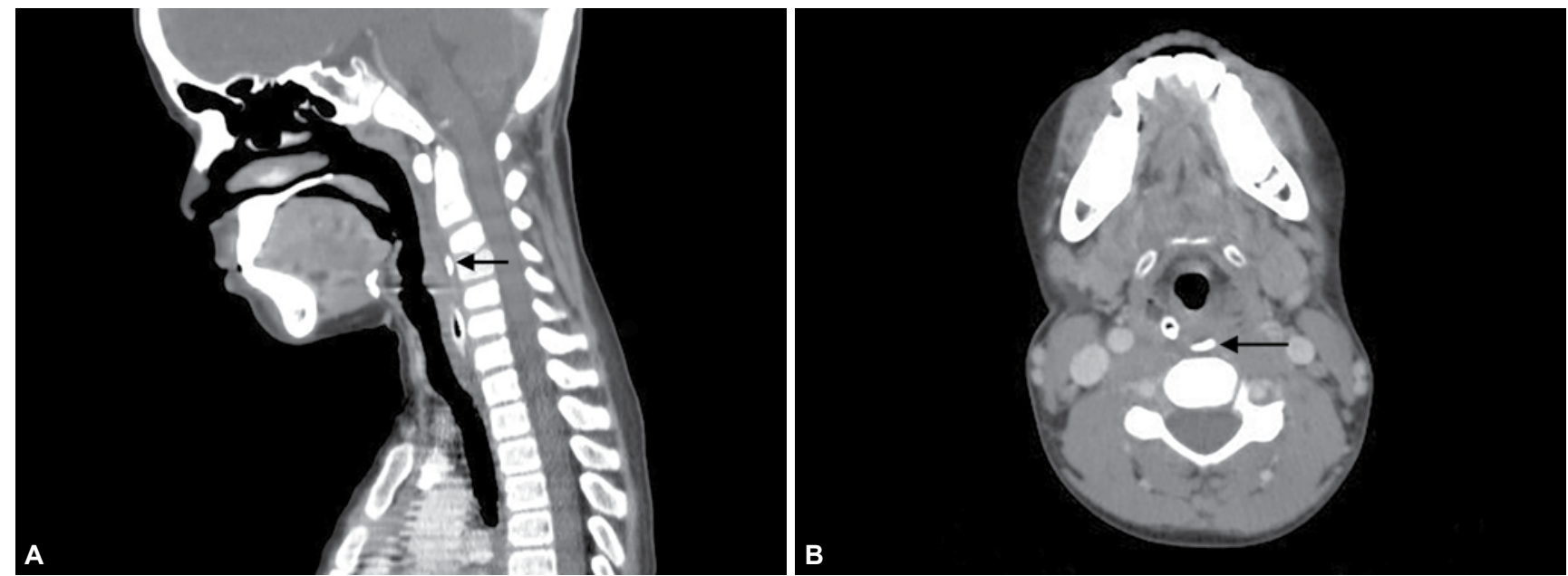

Figs 2A and B: (A) Computed tomography of neck with contrast showing foreign body at level C3-C4 (sagittal) and Ryle's tube seen inferiorly and (B) CT of neck with contrast showing foreign body at level C3-C4 (axial)

He was taken up for re-exploration. As on palpation we could not decide on the region to make the incision, we used image intensifier to guide us. Under fluoroscopic guidance (Figs 3 and 4), foreign body was visualized approximately $8 \mathrm{~mm}$ deep to the mucosal surface of posterior pharyngeal wall, end on, in the sagittal plane against C3-C4 vertebral bodies.

The surgical field was exposed with a direct laryngoscope and the mucosa was incised using a microlaryngeal sickle knife to minimize morbidity. Chicken bone was visualized, and then removed. The thickness of the bone was approximately $2 \mathrm{~mm}$ which probably could be the reason it was not visible or palpable easily. This is where we found image intensifier as a very useful tool in locating the migrated foreign body.

Postoperatively, the patient was started on intravenous antibiotics and nasogastric tube feeds. He symptomatically improved and X-ray soft tissue neck (Fig. 5) taken after 72 hours was normal. He was started on oral feeds and discharged from the hospital.

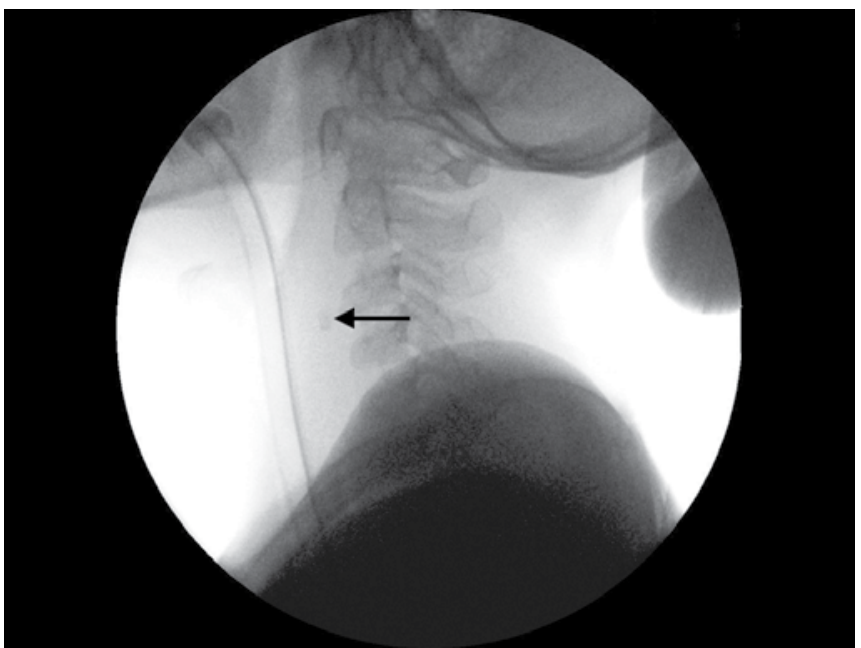

Fig. 3: Fluoroscopy showing foreign body

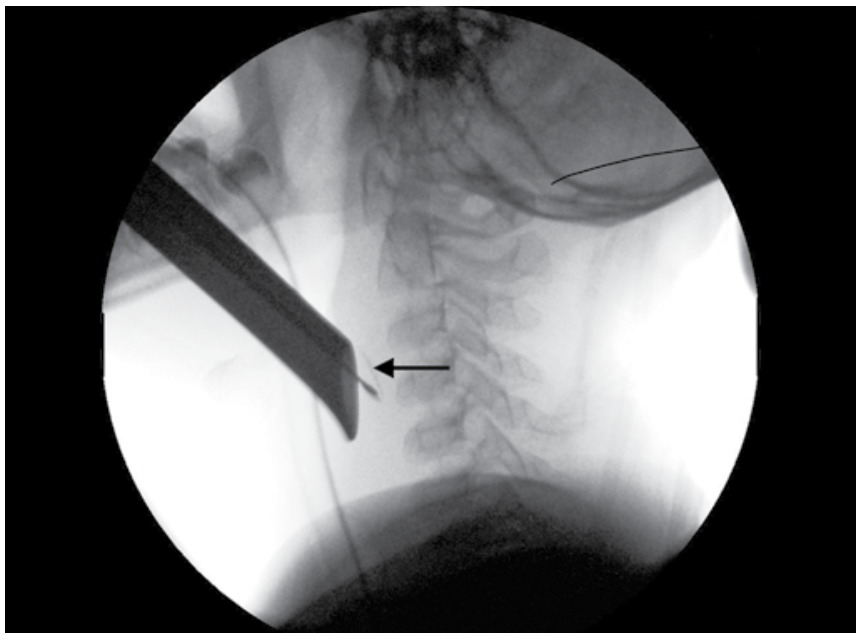

Fig. 4: Fluoroscopy showing foreign body being localized

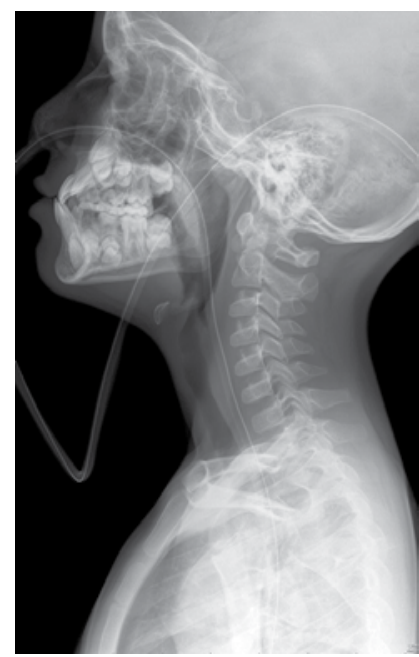

Fig. 5: Postoperative plain radiograph neck lateral

\section{DISCUSSION}

Foreign body ingestion is a common otolaryngological emergency. A clear history of ingestion of sharp objects, such as fish bone or chicken bone, followed by throat pain and difficulty in swallowing, helps us in suspecting an 
impacted foreign body in upper digestive tract. However in children, diagnosis can be challenging because of difficulty in communication. With long standing cases, presenting symptoms may vary depending on the time of ingestion and type of object ingested. Though most foreign bodies get impacted intraluminally, a small percentage can migrate extraluminally. ${ }^{6}$ With time, pressure and reaction to the foreign body, the esophageal wall can perforate. ${ }^{3}$ This is commonly seen with sharp ingested foreign bodies, such as fish bone, dentures or chicken bone. Migration is usually seen to neck and can present as neck swelling or spontaneous extrusion through skin. The movement of neck muscles, esophageal peristalsis probably assist in the movement of these foreign bodies towards neck. ${ }^{6}$ Reports of migration to common carotid artery, thyroid gland and mediastinum are seen in literature. ${ }^{6}$ Delay in diagnosing and managing these conditions can result in life-threatening complications, such as abscess of neck, mediastinitis and also damage to the great vessels. ${ }^{7}$ Removal of migratory foreign bodies is surgically challenging, hence early intervention and management should be planned.

For immediate and accurate diagnosis of impacted foreign body, plain radiograph of neck is very useful. However for radiolucent foreign bodies and in case of long standing history, when suspecting migratory foreign body, better radiological investigation, such as $\mathrm{CT}$ with or without oral contrast will be required. ${ }^{3}$ It helps not only in knowing the exact position of foreign body, but also to decide on the approach for removal. Endoscopic approach is the main modality of diagnosis and removal. Diagnosis of migratory foreign body is made when esophagoscopy is negative in spite of radiograph of neck suggests foreign body. In adults, calcified thyroid cartilage and cricoid cartilage can mimic foreign body.

Neck exploration is recommended to remove migratory foreign body as early as possible in order to avoid life-threatening complications. It carries with it the morbidity of any neck surgery especially in children, along with longer hospital stay, expenditure and also the chance of negative result. To locate a migratory foreign body via endoscopic or open approach can be frustrating to the surgeon. The use of intraoperative imaging, such as image intensifier can be helpful in such difficult cases. ${ }^{3,6}$ In our case, image intensifier assisted us in precise localization and endoscopic removal of foreign body and thereby avoiding a neck exploration.

We report this interesting case to highlight an unusual challenge that an otolaryngologist can face in the management of an ingested foreign body. Optimal use of radiological techniques like CT and fluoroscopy for the precise localization of a migratory foreign body, and thereby facilitating a less radical effective management has been emphasized.

\section{REFERENCES}

1. Shah FA, Reghunandanan NK. A case report of FB (cellular battery) in 18 days old child. Ind J Otolaryngol Head Neck Surg 2011 Oct;63(4):377-379.

2. Peters NJ, Mahajan JK, Bawa M, Chabbra A, Garg R, Rao KLN. Esophageal perforations due to foreign body impaction in children. J Pediatr Surg 2015 Feb 7. pii: S0022-3468(15)00081-0. doi: 10.1016/j.jpedsurg.2015.01.015. [Epub ahead of print]

3. Verma RK, Panda NK. Metallic foreign body migrating into prevertebral muscles: how we did it? Oman Med J 2014 Jan; 29(1):64-66.

4. Uba AF, Sowande AO, Amusa YB, Ogundoyin OO, Chinda JY, Adeyemo AO, et al. Management of oesophageal foreign bodies in children. East Afr Med J 2002 Jun;79(6):334-338.

5. Leong HK, Chan RK. Foreign bodies in the upper digestive tract. Singapore Med J 1987 Apr;28(2):162-165.

6. Bakshi J, Verma RK, Karuppiah S. Migratory foreign body of neck in a battered baby: a case report. Int J Pediatr Otorhinolaryngol 2009 Dec;73(12):1814-1816.

7. Shergill GS, Nayak DR, Dora A, Shergill AK. Migrating foreign bodies in the upper aerodigestive tract: a surgical challenge. BMJ Case Rep 2015 May 20;2015:bcr2015210326. 